\title{
COVID ( 2020-22 )Awareness Control Programme: Brief Review
}

\author{
Jaydip Datta
}

\section{ABSTRACT :}

In this review the awareness control programme, 2020-21 for SARS-COV-2 will review the two publications pointing the interdisciplinary awareness as well as pandemic Covid 19. Corona Virus - a spasmo-demic !. Spasm plus pandemic termed here . spasmodic with respect to our environment. This fatal virological pathogen throughout the world is destroying the environmental sustainability through infectivity rate followed by mortality rate for the last eleven months continuously. The COVID19 ( SARS-COV-2 ) vaccination with successful clinical efficacy trial starting from Europe ( USA , UK ) - U.S.S.R ( Russia ) - Asia ( India

,Pakistan , China ) is the greatest headache for the approval of WHO or successful launching of the vaccines under trial .CITATION - JAYDIP DATTA. (2020, October 6). COVID 19 - An Environmental Pandemic! (Version https://semver.org/spec/v1.0.0-beta.html). http://doi.org/10.17605/OSF.IO/B6DUW . But most important aspect is future awareness program. REF

:https://www.researchgate.net/publication/334836190_INTERDISCIPLINARY_SEMINAR_AND_WORKSHOP_P ARTICIPATION_An_Approach_to_Human_Resource_An_Awareness_Program_against_Covid19_in_near_futur e_FORMERLY_INTERDISCIPLINARY_SEMINAR_AND_WORKSHOP_PARTICIPATION_A_S .

\section{Keywords : Covid20, SARS-COV-2, Human Resource , Pandemic Control , Awareness .}

\section{Full Text References :}

1. JAYDIP DATTA , INFECTION FATALITY RATE OF COVID19 - A LOGISTIC

MODEL ,September 2020 ,DOI:10.31219/osf.io/wuqa6 .

(https://www.researchgate.net/publication/334491992 INFECTION FATALITY RATE OF COVID19 A LOGISTIC MODEL? sg\%5B0\%5D=D3ppSL 4z3DQsha7zLiOUvhw pESRX7YzGEms3sPkMjnk23w a4GjEW0JiNd $v$ EDr-s24Jx4y|RS D4uiHqDMsWMAyAO06khKnlzLeX.mJiquAMod0ssQCglo0SRgnroAwZEAY5loZrhYRjYixRqQcMDwfXhQ VpC24-532ra8-7-LSwsgbDzt4RyRABlkg)

DATTA, J. (2020, September 13). INFECTION FATALITY RATE OF COVID19 - A LOGISTIC MODEL. https://doi.org/10.31219/osf.io/wuqa6 .

DATTA, JAYDIP. "INFECTION FATALITY RATE OF COVID19 - A LOGISTIC MODEL." OSF Preprints, 13 Sept. 2020. Web.

DATTA, JAYDIP. 2020. "INFECTION FATALITY RATE OF COVID19 - A LOGISTIC MODEL." OSF Preprints. September 13. doi:10.31219/osf.io/wuqa6.

2.JAYDIP DATTA. (2020, October 6). COVID 19 -An Environmental Pandemic! (

Version https://semver.org/spec/v1.0.0-beta.html).

http://doi.org/10.17605/OSF.IO/B6DUW .

3.Jaydip Datta, Javier Julian Enriquez, Arash Ziapour, COVID 19 -An Environmental Spasmodemic !, https://www.researchgate.net/publication/340163679.

4.DATTA, JAYDIP (2020): COVID 19 -An Environmental Spasmodemic !. figshare.Preprint. https://doi.org/10.6084/m9.figshare.13456403.v1.

5. DATTA, JAYDIP (2020): INTERDISCIPLINARY SEMINAR AND WORKSHOP PARTICIPATION: An Approach to Human Resource ( HR ( An Awareness Program against Covid19 in near future ). figshare. Presentation.

INTERDISCIPLINARY SEMINAR AND WORKSHOP PARTICIPATION: An Approach to Human Resource ( An Awareness Program against Covid19 in near future ) FORMERLY [INTERDISCIPLINARY SEMINAR AND.. 
7. JAYDIP DATTA. (2020, October 5). COVID19 -A CORRELATION STUDY OF INFECTION FATALITY RATE vs AGE (Version https://semver.org/\#semantic-versioning-200). Zenodo.. http://doi.org/10.5281/zenodo.4066767.

8.JAYDIP DATTA. (2020). Data -SUPPLIMENDARY INFORMATIONS -Growth of D614G Virus Regression Analysis (Version https://semver.org/spec/v1.0.0-beta.html) [Data set]. Zenodo. http://doi.org/10.5281/zenodo.4088751.

9.JAYDIP DATTA. (2020, October 25). CHEMICAL ENGINEERING AND COVID19. Zenodo. http://doi.org/10.5281/zenodo.4129998.

10.JAYDIP DATTA. (2020, November 4). COVID19 DRUG REVIEW DISCUSSIONS (Version Version 1.0). Zenodo. http://doi.org/10.5281/zenodo.4244641.

11. https://www.researchgate.net/post/Vaccinations or antivirals to treat SARS-COV-

2_infection-Which_options_do_you_prefer, JAYDIP DATTA .

12. https://www.researchgate.net/post/Do We belive that spreading of COVIDinfection is an example of aerosol type of Colloid, JAYDIP DATTA.

13.

https://www.researchgate.net/post/How far and how long the world will combat ag ainst mutants of SARS-COV-2, JAYDIP DATTA .

14.

https://www.researchgate.net/post/Whether successive vaccination will increase or decrease HERD IMMUNITY, JAYDIP DATTA .

15. JAYDIP DATTA. (2020). SUPPLIMENTARY Dataset -A STUDY ON STATISTICAL MODELLING ON RECOVERY INFORMATICS OF COVID19 (Version Version 1.0) [Data set]. Zenodo. http://doi.org/10.5281/zenodo.4255246.

16.JAYDIP DATTA. (2020, December 3). Covid19 -A Mathematical modelling of a PSEUDO- GAUSSIUN Contour. Zenodo. http://doi.org/10.5281/zenodo.4304211.

17.JAYDIP DATTA. (2020). Supplementary Data -Indian Covid19 Cases at Different Temperature

(Version https://semver.org/spec/v1.0.0.html\#semantic-versioning-100) [Data set].

18.DATTA, JAYDIP (2020): Covid19 Vaccine development ( ? ) -News update. figshare. Preprint. https://doi.org/10.6084/m9.figshare.12999743.v2 (https://doi.org/16084/m9.figshare.12999743.v2 ).

19.DATTA, JAYDIP (2020): COVID 19 - An Environmental Spasmodemic !. figshare. Preprint. https://doi.org/10.6084/m9.figshare.13456403.v1 (https://doi.org/10.6084/m9.figshare.13456403.v1).

20. DATTA, JAYDIP (2020): INTERDISCIPLINARY SEMINAR AND WORKSHOP PARTICIPATION: An Approach to Human Resource ( HR ( An Awareness Program against Covid19 in near future ). figshare. Presentation.

https://doi.org/10.6084/m9.figshare.12295529.v4 (https://doi.org/10.6084/m9.figshare.12295529.v4).

21.JAYDIP DATTA -https://www.researchgate.net/post/Corona-Pedagogy-

Both in Teaching and Research(

, $31^{\text {st }}$ Jan ,2020).

22.JAYDIP DATTA. (2021, January 3). COVID ( 2020-21 )Awareness Control Programme: BriefReview

(Version https://semver.org/spec/v1.0.0-beta.html). Zenodo. http://doi.org/10.5281/zenodo.4412246. 23..Jaydip Datta , Ehsan Mohammed Abdelgadir, PRIMARY HEALTH CARE AND PEACE : ANOTHER WAY OF SUSTAINABLE ENVIRONMENT ( Experiment Finding )- Addendum for Covid 19 , September 2019 ,DOI : 10.17605/OSF.IO/BPXN2 .

(https://www.researchgate.net/publication/335587570 PRIMARY HEALTH CARE AND PEACE ANOTHER WAY OF SUSTAINABLE ENVIRONMENT Experiment Finding -

Addendum for Covid 19? sg=O9cF0wEJczo3Tyv1SEzai5WgJl-Ovp3rg350SHCvOfVV-

ka 9QgPJYCt0CtzznzxLqeKyqcHvcMIDSUQEEY8AHnQD7dEIYQW2aTCaZN .1aNuniDR- 
) .

24. JAYDIP DATTA ,A SEMINAR ON ARSENIC AS A KEY WATER POLLUTANTS , December 2006 ,DOI: $\underline{10.13140 / R G .2 .2 .17913 .31849}$.

(

https://www.researchgate.net/publication/333974066 A SEMINAR ON ARSENIC AS A KEY WATER POLLU TANTS? sg=SJ6 3hkcL2r5 NqQeREG87VLvmyX9fEYqGkh7QHfsWrhbNdnGzodzRGnil|A-jVzuy-IDEnahnHzor5s9mSUg5-VoLKb5XAdMV-

WUuh6.XLzx8ffH1UZrExviSE8zeh1u37wG1R5Ypp4cLTPduFakLFq0m Zrarrhure1ZLubmrALIXPQapKVkMRZi20 PXA)

Pandemic Control - To conclude from the view of Human Resource (HR ) training / workshop / Seminar participation is a Key functioning area . In this note I am presenting the major issues of HR namely .Environment \& Public Health Management to eradicate Corona Virus after lockdown - period to develop a sharp awareness program against such epidemic / pandemic situations ( The Do or Don'ts ). Here resource persons are Doctors , Common public, Health workers \& patients affected with Covid19 \& administration ie GOVT with police. Apart from them humanities, scientific people, economists are also the resource persons. In the Concluding session we may hope that introduction of this type of INTERDISCIPLINARY SEMINARS as specified the above areas with PARTICIPATION OF THE RESOURCE PEOPLE will definitely combat against such PANDEMIC situation or a GOOD MANAGEMENT OF EPIDEMOLOGY IN NEAR FUTURE .REF INTERDISCIPLINARY SEMINAR AND WORKSHOP PARTICIPATION: An Approach to Human Resource ( An Awareness Program against Covid19 in near future ) FORMERLY [INTERDISCIPLINARY SEMINAR AND... Preprint • May 2020 DOI:

10.13140/RG.2.2.24412.33926/2 https://www.researchgate.net/publication/334836190. 